\title{
SSM-DENCLUE: Enhanced Approach for Clustering of Sequential Data: Experiments and Test Cases
}

\author{
K.Santhi Sree, Ph.D \\ Professor of CSE \\ JawaharLal Nehru Technological University \\ Kukatpally, Hyderabad
}

\begin{abstract}
Clustering web usage data is useful to discover interesting patterns related to user traversals, behavior and their characteristics, which helps for the improvement of better Search Engines and Web personalization. Clustering web sessions is to group them based on similarity and consists of minimizing the Intra-cluster similarity and maximizing the Inter-group similarity. The other issue that arises is how to measure similarity between web sessions. There exist multiple similarity measures in the past like Euclidean, Jaccard ,Cosine and many. Most of the similarity measures presented in the history deal only with sequence data but not the order of occurrence of data. A novel similarity measure named $\operatorname{SSM}$ (Sequence Similarity Measure) is developed that shows the impact of clustering process, when both sequence and content information is incorporated while computing similarity between sequences. SSM (Sequence Similarity measure) captures both the order of occurrence of page visits and the page information as well , and compared the results with Euclidean, Jaccard and Cosine similarity measures. Incorporating a new similarity measure, the existing Density clustering technique DENCLUE is enhanced and the new named as SSM-DENCLUE for Web personalization. The Inter-cluster and Intra-cluster distances are computed using Average Levensthien distance (ALD) to demonstrate the usefulness of the proposed approach in the context of web usage mining. This new similarity measure has significant results when comparing similarities between web sessions with other previous measures, and provided good time requirements of the newly developed SSM- DENCLUE algorithms. Experiments are performed on MSNBC.COM website ( free online news channel), in the context of Density based clustering in the domain of Web usage mining.
\end{abstract}

\section{General Terms}

Sequence Mining, Clustering .Density Based Clustering

\section{Keywords}

Data Mining, Clustering, similarity measures, Web Personalization, DENCLUE, SSM-DENCLUE.

\section{INTRODUCTION}

\subsection{Data Mining}

Data mining, called Knowledge Discovery in Databases(KDD) an interdisciplinary subfield of computer science is the process of identifying knowledge / patterns in large heterogeneous data sets. The goal of the Data mining process is to extract information from a data set, preprocess and transform it into an understandable structure for further use. Various stages of Data mining are Selection, Preprocessing , Transformation , Data mining, Interpretation and evaluation. The various Data mining techniques are
Classification, Clustering, Prediction, Association and Discrimination.

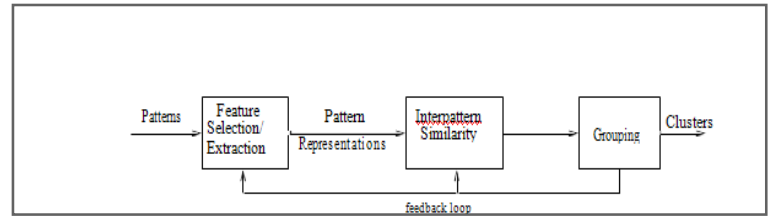

Figure 1: Data Mining Architecture

\subsection{Clustering}

Is a process of categorizing the data into multiple clusters where all the patterns lying in one cluster are similar to one another and dissimilar when compared to the patterns lying in the other cluster. Different types of clustering techniques are partitioning, Hierarchical, Densitybased ,Grid-based and Model-Based algorithms. In Density-based clustering, clusters are defined as areas of higher density than the remainder of the dataset.The most popular Density based clustering method is DBSCAN, OPTICS and DENCLUE. They start with an arbitrary starting point $\mathrm{P}$ that has not been visited and its e-neighborhood is retrieved, and if it contains sufficient number of points, a cluster is started. Otherwise, the point is labeled as noise.

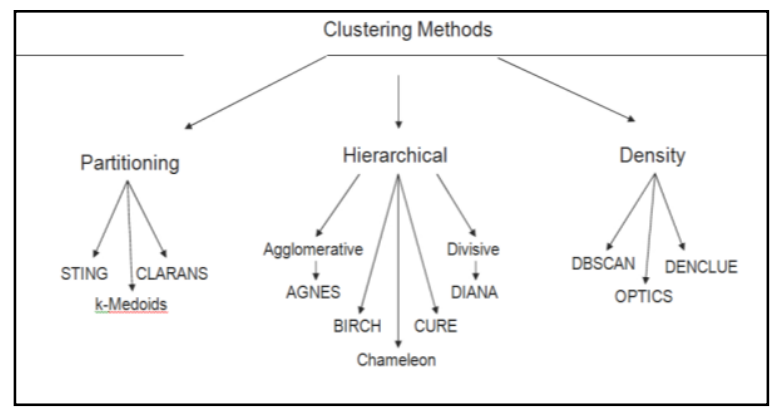

Figure 2: Types of clustering techniques

\subsection{Sequence Mining}

Sequential Pattern mining is interdisciplinary subfield of Data mining concerned with finding relevant patterns described in a sequence. Given a sequence database $\mathrm{D}=\{\mathrm{S} 1, \mathrm{~S} 2, \ldots, \mathrm{Sn}\}$ where each sequence $\mathrm{S}$ is an ordered list of events/items <i1, i2,...,in>.There are several key traditional computational problems addressed within this field. These include building efficient databases and indexes for sequence information, extracting the frequently occurring patterns, comparing sequences for similarity, and recovering missing sequence numbers. In general, sequence mining problems can be classified as string mining which is typically based 
on string based algorithms and itemset mining which is typically based on association rule mining.

\subsection{Web Personalization}

Web personalization is the process of identifying what users are exactly looking for on the web, their traversals and their behavior. Due to the continuous growth of the Web data, Web personalization has become one of the challenging task for the researchers and commercial areas. The steps of a Web personalization process include: the collection of Web data, modeling and categorization of these data (preprocessing phase), the analysis of the collected data, the determination of the actions that should be performed. Web data are collected and used in the context of Web personalization. These data are classified in four categories .web Structure data represent how pages are linked to one another. Web usage data represents what users are exactly looking for on the Web and their characteristics such as a visitor's IP address, time and date of access, complete path (files or directories) accessed, referrers' address, and other attributes that can be included in a Web access log.

\subsection{Similarity Measures}

Similarity measure are used to find out how similar are two sequences are. In the history many similarity measures exist, and they are Euclidean, Jaccard, Cosine, Manhanttan and Minkowski measures. These similarity measures are vector based. Euclidean distance measure is frequency based similarity measures for two sequences $S_{1}$ and $S_{2}$ in an $\mathrm{N}$ - dimensional space. It is defined as the square root of the sum of the corresponding dimensions of the vector. The Euclidean distance between sequences $\mathrm{S}_{1}=\left(p_{1}, p_{2}, \ldots, p_{n}\right)$ and $\mathrm{S}_{2}=\left(q_{1}, q_{2}, \ldots, q_{n}\right)$ is defined as

$$
\begin{aligned}
& \operatorname{Sim}\left(S_{1}, S_{2}\right) \\
& =\sqrt{\left(S_{1_{1}}-S_{2_{1}}\right)^{2}+\left(S_{1_{2}}-S_{2_{2}}\right)^{2}+\cdots+\left(S_{1_{n}}-S_{2_{n}}\right)^{2}} \\
& =\sqrt{\sum_{i=1}^{n}\left(S_{1_{i}}-S_{2_{i}}\right)^{2}}
\end{aligned}
$$

Jaccard similarity measure is defined as the ratio of the intersection of items between the two sequences to the union of items of the two sequences.

$$
\left(\operatorname{Sim}\left(S_{1}, S_{2}\right)\right)=\frac{\boldsymbol{S}_{1} \boldsymbol{S}_{2}}{\left|\boldsymbol{S}_{1}\right|^{2}+\left|\boldsymbol{S}_{2}\right|^{2}-\boldsymbol{S}_{1} \boldsymbol{S}_{\mathbf{2}}}
$$

Cosine similarity measure is the angle between two vectors. The cosine measure is given by

$$
\operatorname{Sim}\left(S_{1}, S_{2}\right)=\frac{\sum_{i=1}^{n}\left(\boldsymbol{S}_{1} \times \boldsymbol{S}_{2}\right)}{\sqrt{\sum_{i=1}^{n}\left(\boldsymbol{S}_{\mathbf{1}_{i}}\right)^{2}} \times \sqrt{\sum_{i=1}^{n}\left(\boldsymbol{S}_{\mathbf{2}_{i}}\right)^{2}}}
$$

\subsection{SSM-Sequence Similarity Measure}

In this work a novel similarity measure [2] is used that captures both the order of information as well as content(information) called the $\operatorname{SSM}$ ( sequence similarity measure).

$$
\begin{aligned}
\operatorname{SSM}\left(S_{1}, S_{2}\right)=\frac{S_{1}}{S_{1}} \cup S_{2} & S_{2} * \operatorname{FC}\left(S_{1}, S_{2}\right) \\
& +\frac{\operatorname{LLCS}\left(S_{1}, S_{2}\right)}{\sqrt{\sum_{i=1}^{n}\left(S_{1_{i}}\right)^{2}} \times \sqrt{\sum_{i=1}^{n}\left(S_{2_{i}}\right)^{2}}}
\end{aligned}
$$

\section{EXISTING METHODOLOGY}

Usually when dealing with sequences, the data is converted into n-dimensional frequency vectors. The vector representation can be either indicating presence or absence of symbol in a sequence, or, indicating frequency of symbol within a sequence. While computing similarity between sequences they either consider the content /information or the order information. In the existing work the sequences are converted to intermediate representations and the similarity between any two sequences is calculated using any of the similarity measures like Euclidean, Jaccard, Cosine. DENCLUE can be applied for clustering. Similarity are calculated which illustrates the similarity between the sequences. And the Inter cluster similarity has to be maximized and Intra cluster similarity has to be minimized.



Figure 3: Exiting Work Procedure

\section{PROPOSED WORK}

The work concentrates on Clustering technique on the domain of web usage data. A new similarity measure [6] is used to measure similarity/distance between two sequences and experiments are conducted on Density -based clustering using existing clustering called DENCLUE and the enhanced Density clustering algorithm like SSM-DENCLUE respectively .In all the experiments the running time of the new similarity measure is as accurate as the best compared to the earlier similarity measures . An experimental framework for sequential data stream mining on clustering on web usage data is built.

\subsection{Experimental Results}

\subsubsection{Web Navigation dataset used for Testing}

MSNBC is a joint venture between Microsoft and NBC(National Broad casting) is a famous online news website with has different news subjects. There are 17 categories of news like frontpage,news,tech,local,opinion,onair, weather,health,living, business,sports,summary,bbs,travelmisc,msn-news and msnsports. For example, 'frontpage' is coded as 1, 'news' as 2, 
'tech' as 3, etc. Web Navigational dataset is considered in Table 5.1

Table 1. Web Navigational Dataset

\begin{tabular}{|l|l|}
\hline T1 & on-air misc misc misc on-air misc \\
\hline T2 & news sports tech local sports ,sports \\
\hline T3 & Sports bbs bbs bbs bbs bbs bbs \\
\hline T4 & frontpage frontpage sports news news local \\
\hline T5 & on-air weather weather weather sports,sports \\
\hline T6 & on-air on-air on-air on-air tech bbs \\
\hline T7 & frontpage bbs bbs frontpage frontpage news \\
\hline T8 & $\begin{array}{l}\text { frontpage frontpage frontpage frontpage frontpage } \\
\text { bbs }\end{array}$ \\
\hline T9 & news news travel opinion opinion msn-news \\
\hline T10 & frontpage business frontpage news news bbs \\
\hline
\end{tabular}

Table 2. Converted Web Navigational dataset

\begin{tabular}{|l|l|}
\hline Sequence & \multicolumn{1}{|c|}{ Order of page visists } \\
\hline T1 & $6,15,15,15,6,15$ \\
\hline T2 & $2,11,3,4,11,11$ \\
\hline T3 & $11,13,13,13,13,13$ \\
\hline T4 & $1,1,11,2,2,4$ \\
\hline T5 & $6,7,7,7,11,11$ \\
\hline T6 & $6,6,6,6,3,6$ \\
\hline T7 & $1,13,13,1,1,2$ \\
\hline T8 & $1,1,1,1,1,13$ \\
\hline T9 & $2,2,14,5,5,16$ \\
\hline T10 & $1,10,1,2,2,13$ \\
\hline
\end{tabular}

\subsection{DENCLUE and SSM-DENCLUE Clustering Technique}

\subsubsection{Experiments on Synthetic web Navigational}

\section{Dataset for DENCLUE}

Consider arbitrarily 200 records of web transactions from MSNBC.COM website. The transactions are converted to vector representation, and a $200 \times 200$ similarity matrix is computed using any of the existing similarity measures mentioned above. In the step two after applying existing Denclue clustering technique the clusters formed are 17. Table 7.4, $17 \times 17$ matrix which shows the inter cluster distance using Euclidean distance measure. For example, the inter cluster distance $(\mathrm{C} 1, \mathrm{C} 2)=0.15$. and inter cluster distance between the clusters $(\mathrm{C} 3, \mathrm{C} 16)=0.31$. That is the patterns lying in the cluster $\mathrm{C} 3$ are more dissimilar when compared to the patterns lying in the cluster $\mathrm{C} 16$

Table 3: Inter Cluster Distance Using Euclidean Distance Measure for Denclue

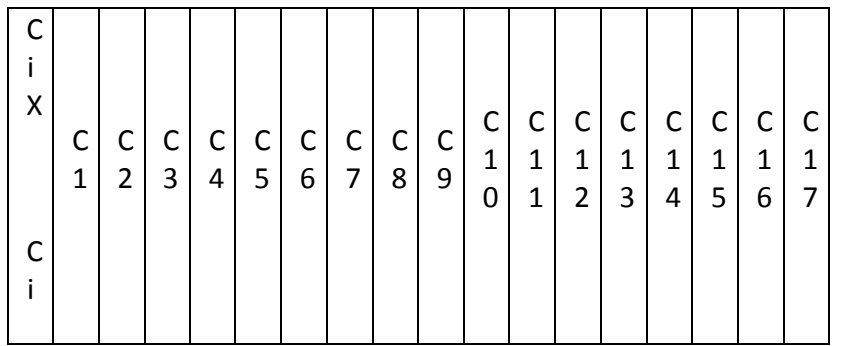

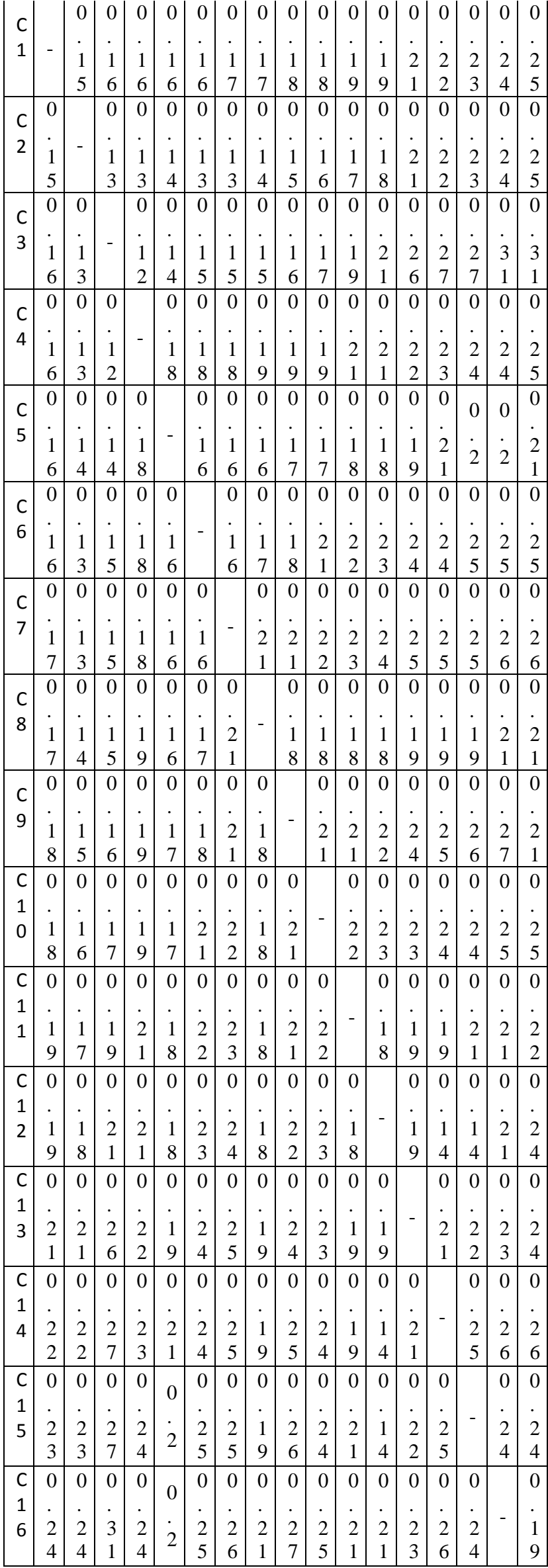


\begin{tabular}{|l|l|l|l|l|l|l|l|l|l|l|l|l|l|l|l|l|l|}
0 & 0 & 0 & 0 & 0 & 0 & 0 & 0 & 0 & 0 & 0 & 0 & 0 & 0 & 0 & 0 & 0
\end{tabular} $\begin{array}{lllllllllllllllll}1 & \dot{2} & \mathbf{2} & 3 & \mathbf{2} & \dot{2} & \mathbf{2} & \mathbf{2} & \mathbf{2} & \dot{2} & \mathbf{2} & \mathbf{2} & \mathbf{2} & \dot{2} & \mathbf{2} & \mathbf{2} & 1\end{array}$ \begin{tabular}{llllllllllllllllll|l|l|l|l}
7 & 5 & 5 & 1 & 5 & 1 & 5 & 6 & 1 & 1 & 5 & 2 & 4 & 4 & 6 & 4 & 9
\end{tabular}

Table 4: Inter Cluster Distance Using Jaccard Distance Measure For Denclue

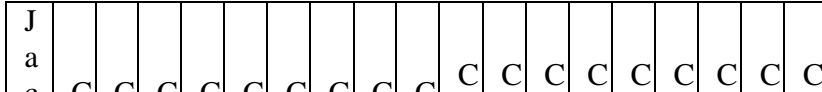
\begin{tabular}{lllllllllllllllllllllll}
$\mathrm{c}$ & $\mathrm{C}$ & $\mathrm{C}$ & $\mathrm{C}$ & $\mathrm{C}$ & $\mathrm{C}$ & $\mathrm{C}$ & $\mathrm{C}$ & $\mathrm{C}$ & $\mathrm{C}$ & $\mathrm{C}$ & $\mathrm{C}$ & $\mathrm{C}$ & $\mathrm{C}$ & $\mathrm{C}$ & $\mathrm{C}$ & $\mathrm{C}$ & $\mathrm{C}$ & $\mathrm{C}$ \\
\hline & & & &
\end{tabular} $\begin{array}{lllllllllllllllllllllll}\mathrm{c} & 1 & 2 & 3 & 4 & 5 & 6 & 7 & 8 & 9 & 1 & 1 & 1 & 1 & 1 & 1 & 1 & 1 & 1 \\ 0 & 1 & 2 & 3 & 4 & 5 & 6 & 7 & 8\end{array}$ a

d

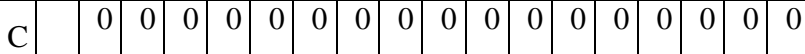


\begin{tabular}{llllllllllllllllllllll}
5 & 6 & 6 & 6 & 6 & 7 & 7 & 8 & 8 & 9 & 9 & 1 & 2 & 3 & 4 & 5 & 5 \\
\hline
\end{tabular} $\begin{array}{llllllllllllllllllll}\mathrm{C} & 0 & & 0 & 0 & 0 & 0 & 0 & 0 & 0 & 0 & 0 & 0 & 0 & 0 & 0 & 0 & 0 & 0\end{array}$ $\begin{array}{llllllllllllllllllll}2 & \dot{1} & 0 & \dot{1} & \dot{1} & \dot{1} & \dot{1} & \dot{1} & \dot{1} & \dot{1} & \dot{1} & \dot{1} & \dot{1} & \dot{2} & \dot{2} & \dot{2} & \dot{2} & \dot{2} & \dot{2} \\ & 1 & & & 1 & & & \end{array}$ \begin{tabular}{llllllllllllllllllllll}
5 & & 3 & 3 & 4 & 3 & 3 & 4 & 5 & 6 & 7 & 8 & 1 & 2 & 3 & 4 & 5 & 6 \\
\hline
\end{tabular} $\begin{array}{llllllllllllllllllll}\mathrm{C} & 0 & 0 & & 0 & 0 & 0 & 0 & 0 & 0 & 0 & 0 & 0 & 0 & 0 & 0 & 0 & 0 & 0\end{array}$

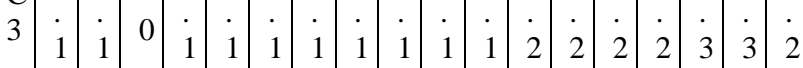
\begin{tabular}{lllllllllllllllllllllll}
6 & 3 & & 2 & 4 & 5 & 5 & 5 & 6 & 7 & 9 & 1 & 6 & 7 & 7 & 1 & 1 & 9 \\
\hline
\end{tabular}

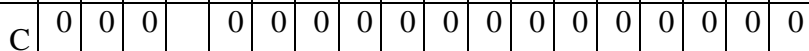


\begin{tabular}{lllllllllllllllllllll}
6 & 3 & 2 & & 8 & 8 & 8 & 9 & 9 & 9 & 1 & 1 & 2 & 3 & 4 & 4 & 5 & 6 \\
\hline
\end{tabular} C \begin{tabular}{lllllllllllllllllll}
$\mathrm{C}$ & 0 & 0 & 0 & & 0 & 0 & 0 & 0 & 0 & 0 & 0 & 0 & 0 & 0 & 0 & 0 & 0 \\
\hline
\end{tabular}

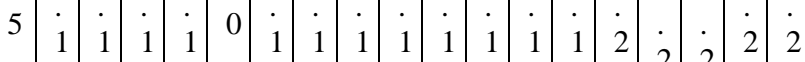

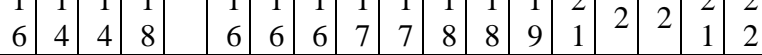
$\begin{array}{llllllllllllllllllllll}\mathrm{C} & 0 & 0 & 0 & 0 & 0 & & 0 & 0 & 0 & 0 & 0 & 0 & 0 & 0 & 0 & 0 & 0 & 0\end{array}$ $\begin{array}{lllllllllllllllllll}6 & \dot{1} & \dot{1} & 1 & \dot{1} & \dot{1} & 0 & \dot{1} & \dot{1} & \dot{1} & \dot{2} & \dot{2} & \dot{2} & \dot{2} & \dot{2} & \dot{2} & \dot{2} & \dot{2} & \dot{2} \\ & 6 & & & & 8 & 6 & & 6 & & \end{array}$ $\begin{array}{lllllllllllllllllllllll}6 & 3 & 5 & 8 & 6 & & 6 & 7 & 8 & 1 & 2 & 3 & 4 & 4 & 5 & 5 & 5 & 5\end{array}$

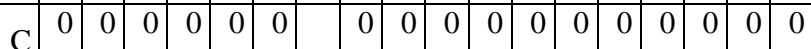
$\begin{array}{lllllllllllllllllll}7 & \dot{1} & 1 & 1 & 1 & 1 & 1 & 0 & \dot{2} & \dot{2} & 2 & \dot{2} & \dot{2} & 2 & \dot{2} & \dot{2} & 2 & \dot{2} & \dot{2}\end{array}$ \begin{tabular}{lllllllllllllllllllllll}
7 & 3 & 5 & 8 & 6 & 6 & & 1 & 1 & 2 & 3 & 4 & 5 & 5 & 5 & 6 & 6 & 7 \\
\hline
\end{tabular}


$\begin{array}{lllllllllllllllllll}8 & \dot{1} & \dot{1} & \dot{1} & \dot{1} & \dot{1} & \dot{1} & \dot{2} & 0 & \dot{1} & \dot{1} & \dot{1} & \dot{1} & \dot{1} & \dot{1} & \dot{1} & \dot{2} & \dot{2} & \dot{2} \\ & 7 & 4 & & & & & & & \end{array}$ \begin{tabular}{llllllllllllllllllll}
7 & 4 & 5 & 9 & 6 & 7 & 1 & & 8 & 8 & 8 & 8 & 9 & 9 & 9 & 1 & 1 & 2 \\
\hline
\end{tabular} $\mathrm{C}$
$\mathrm{C}$ $\begin{array}{lllllllllllllllllll}9 & \dot{1} & \dot{1} & 1 & \dot{1} & \dot{1} & \dot{1} & \dot{2} & \dot{1} & 0 & \dot{2} & \dot{2} & \dot{2} & \dot{2} & \dot{2} & \dot{2} & \dot{2} & \dot{2} & \dot{2} \\ & 8 & 5 & 6 & 9 & 7 & 8 & & \end{array}$ $\begin{array}{llllllllllllllllllllllll}8 & 5 & 6 & 9 & 7 & 8 & 1 & 8 & & 1 & 1 & 2 & 4 & 5 & 6 & 7 & 1 & 2\end{array}$

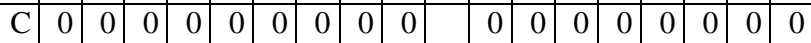
$\begin{array}{lllllllllllllllllll}1 & \dot{1} & \dot{1} & 1 & \dot{1} & \dot{1} & \dot{2} & \dot{2} & \dot{1} & \dot{2} & 0 & \dot{2} & \dot{2} & \dot{2} & \dot{2} & \dot{2} & \dot{2} & \dot{2} & \dot{2} \\ 0 & 1 & & & & \end{array}$ \begin{tabular}{lllllllllllllllllllllll}
8 & 6 & 7 & 9 & 7 & 1 & 2 & 8 & 1 & & 2 & 3 & 3 & 4 & 4 & 5 & 5 & 7 \\
\hline
\end{tabular}


\begin{tabular}{llllllllllllllllllllll}
1 & $\dot{1}$ & $\dot{1}$ & 1 & $\dot{2}$ & $\dot{1}$ & $\dot{2}$ & $\dot{2}$ & $\dot{1}$ & $\dot{2}$ & $\dot{2}$ & 0 & $\dot{1}$ & $\dot{1}$ & $\dot{1}$ & $\dot{2}$ & $\dot{2}$ & $\dot{2}$ & $\dot{2}$ \\
\hline
\end{tabular} \begin{tabular}{lllllllllllllllllllllll}
1 & 1 & 1 & 2 & 1 & 2 & 2 & 1 & 2 & 2 & 0 & 1 & 1 & 1 & 2 & 2 & 2 & 2 \\
9 & 7 & 9 & 1 & 8 & 2 & 3 & 8 & 1 & 2 & & 8 & 9 & 9 & 1 & 1 & 2 & 2 \\
\hline
\end{tabular} \begin{tabular}{lllllllllllllllllllllll}
$\mathrm{C}$ & 0 & 0 & 0 & 0 & 0 & 0 & 0 & 0 & 0 & 0 & 0 & & 0 & 0 & 0 & 0 & 0 & 0 \\
\hline
\end{tabular} \begin{tabular}{llllllllllllllllllll}
1 & $\dot{1}$ & $\dot{1}$ & $\dot{2}$ & $\dot{2}$ & $\dot{1}$ & $\dot{2}$ & $\dot{2}$ & $\dot{1}$ & $\mathbf{2}$ & $\dot{2}$ & $\dot{1}$ & 0 & $\dot{1}$ & $\dot{1}$ & $\dot{1}$ & $\dot{2}$ & $\dot{2}$ & 2 \\
\hline
\end{tabular} $\begin{array}{lllllllllllllllllllll}9 & 8 & 1 & 1 & 8 & 3 & 4 & 8 & 2 & 3 & 8 & & 9 & 4 & 4 & 1 & 4 & 4\end{array}$ \begin{tabular}{lllllllllllllllllll|}
$\mathrm{C}$ & 0 & 0 & 0 & 0 & 0 & 0 & 0 & 0 & 0 & 0 & 0 & 0 & 0 & 0 & 0 & 0 & 0 & 0 \\
\hline
\end{tabular} \begin{tabular}{|l|l|l|l|l|l|l|l|l|l|l|l|l|l|l|l|l|l|l|}
\hline 3 & 2 & 2 & 2 & 2 & 1 & 2 & 2 & 1 & 2 & 2 & 1 & 1 & & 2 & 2 & 2 & 2 & 2 \\
\hline
\end{tabular} \begin{tabular}{lllllllllllllllllllllllll}
1 & 1 & 6 & 2 & 9 & 4 & 5 & 9 & 4 & 3 & 9 & 9 & & 1 & 2 & 3 & 4 & 5 \\
\hline & 0 & 0 & 0 & 0 & 0 & 0 & 0 & 0 & 0 & 0 & 0 & 0 & 0 & & 0 & 0 & 0 & 0
\end{tabular} \begin{tabular}{llllllllllllllllllll}
$\mathrm{C}$ & 0 & 0 & 0 & 0 & 0 & 0 & 0 & 0 & 0 & 0 & 0 & 0 & 0 & & 0 & 0 & 0 & 0 \\
\hline
\end{tabular} $\begin{array}{lllllllllllllllllll}1 & \dot{2} & \dot{2} & \dot{2} & \dot{2} & \dot{2} & \dot{2} & \dot{2} & \dot{1} & \dot{2} & \dot{2} & \dot{1} & \dot{1} & \dot{2} & 0 & \dot{2} & \dot{2} & \dot{2} & \dot{2}\end{array}$ $\begin{array}{llllllllllllllllllllll}2 & 2 & 7 & 3 & 1 & 4 & 5 & 9 & 5 & 4 & 9 & 4 & 1 & & 5 & 6 & 6 & 7\end{array}$

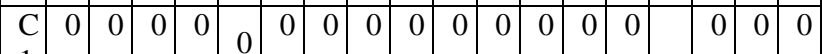

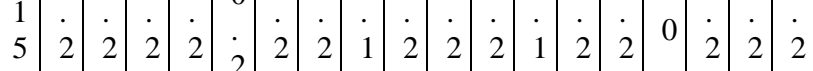
\begin{tabular}{lllllllllllllllllllll}
5 & 2 & 2 & 2 & 2 & & 2 & 2 & 1 & 2 & 2 & 2 & 1 & 2 & 2 & 0 & 2 & 2 & 2 \\
& 3 & 3 & 7 & 4 & 2 & 5 & 5 & 9 & 6 & 4 & 1 & 4 & 2 & 5 & & 4 & 4 & 4 \\
\hline
\end{tabular} $\begin{array}{lllllllllllllllllllll}\mathrm{C} & 0 & 0 & 0 & 0 & 0 & 0 & 0 & 0 & 0 & 0 & 0 & 0 & 0 & 0 & 0 & & 0 & 0\end{array}$ \begin{tabular}{llllllllllllllllllllll}
1 & 6 & 2 & 2 & 3 & 2 & $\dot{2}$ & 2 & 2 & 2 & 2 & 2 & 2 & 2 & $\dot{2}$ & 2 & $\dot{2}$ & 0 & $\dot{1}$ & 2 \\
\hline
\end{tabular} \begin{tabular}{llllllllllllllllllllll}
4 & 4 & 1 & 4 & 2 & 5 & 6 & 1 & 7 & 5 & 1 & 1 & 3 & 6 & 4 & & 9 & 3 \\
\hline
\end{tabular}

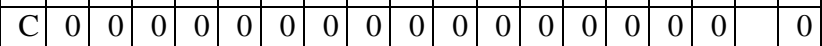
$\begin{array}{lllllllllllllllllll}7 & \dot{2} & 2 & \dot{3} & \dot{2} & \dot{2} & \dot{2} & \dot{2} & \dot{2} & \dot{2} & \dot{2} & \dot{2} & \dot{2} & \dot{2} & \dot{2} & \dot{2} & \dot{1} & 0 & \dot{1}\end{array}$ \begin{tabular}{llllllllllllllllllll}
5 & 5 & 1 & 5 & 1 & 5 & 6 & 1 & 1 & 5 & 2 & 4 & 4 & 6 & 4 & 9 & & 9 \\
\hline & 0 & 0 & 0 & 0 & 0 & 0 & 0 & 0 & 0 & 0 & 0 & 0 & 0 & 0 & 0 & 0 & 0 &
\end{tabular}

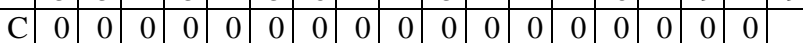

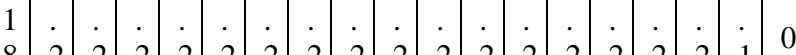
$\begin{array}{lllllllllllllllllll}8 & 2 & 2 & 2 & 2 & 2 & 2 & 2 & 2 & 2 & 2 & 2 & 2 & 2 & 2 & 2 & 2 & 1 & 0 \\ & 5 & 6 & 9 & 6 & 2 & 5 & 7 & 2 & 2 & 7 & 2 & 4 & 5 & 7 & 4 & 3 & 9\end{array}$

Table 5: Inter Cluster Distance Using Cosine Similarity Measure For Denclue

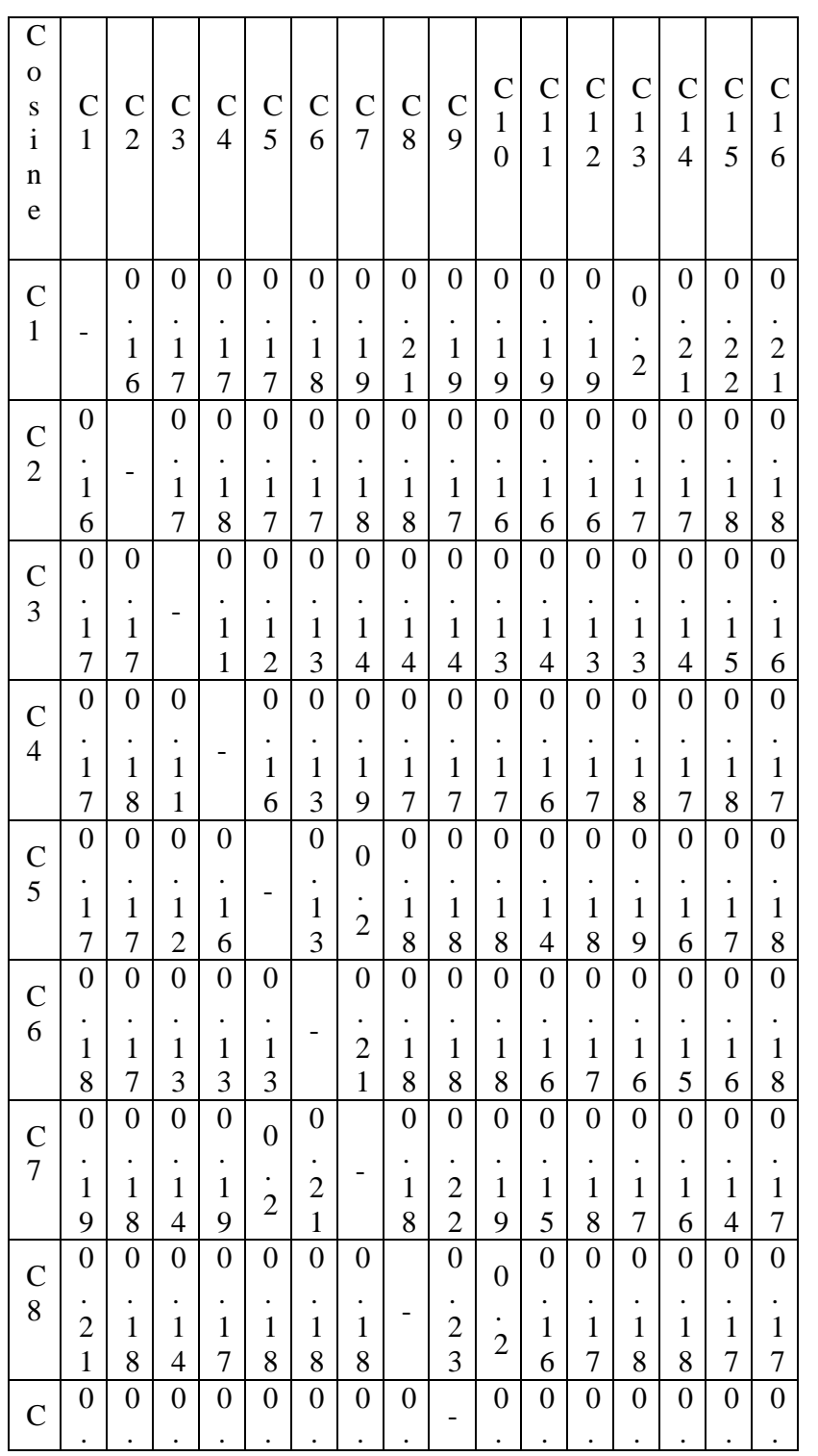




\begin{tabular}{|l|l|l|l|l|l|l|l|l|l|l|l|l|l|l|l|l|}
\hline 9 & 1 & 1 & 1 & 1 & 1 & 1 & 2 & 2 & & 2 & 2 & 1 & 1 & 1 & 2 & 1 \\
& 9 & 7 & 4 & 7 & 8 & 8 & 2 & 3 & & 1 & 1 & 7 & 8 & 6 & 1 & 8 \\
\hline $\mathrm{C}$ & 0 & 0 & 0 & 0 & 0 & 0 & 0 & 0 & 0 & & 0 & 0 & 0 & 0 & 0 & 0 \\
1 &. &. &. &. &. &. &. &. &. & - &. &. &. &. &. &. \\
0 & 1 & 1 & 1 & 1 & 1 & 1 & 1 &. & 2 & - & 2 & 1 & 1 & 1 & 2 & 1 \\
& 9 & 6 & 3 & 7 & 8 & 8 & 9 & 2 & 1 & & 2 & 9 & 9 & 7 & 2 & 8 \\
\hline $\mathrm{C}$ & 0 & 0 & 0 & 0 & 0 & 0 & 0 & 0 & 0 & 0 & & 0 & 0 & 0 & 0 & 0 \\
1 &. &. &. &. &. &. &. &. &. &. & - &. &. &. &. &. \\
1 & 1 & 1 & 1 & 1 & 1 & 1 & 1 & 1 & 2 & 2 & & 2 & 2 & 2 & 2 & 2 \\
& 9 & 6 & 4 & 6 & 4 & 6 & 5 & 6 & 1 & 2 & & 7 & 3 & 3 & 2 & 4 \\
\hline $\mathrm{C}$ & 0 & 0 & 0 & 0 & 0 & 0 & 0 & 0 & 0 & 0 & 0 & & 0 & 0 & 0 & 0 \\
1 &. &. &. &. &. &. &. &. &. &. &. & &. &. &. &. \\
2 & 1 & 1 & 1 & 1 & 1 & 1 & 1 & 1 & 1 & 1 & 2 & - & 1 & 1 & 1 & 1 \\
& 9 & 6 & 3 & 7 & 8 & 7 & 8 & 7 & 7 & 9 & 7 & & 8 & 7 & 8 & 7 \\
\hline $\mathrm{C}$ & 0 & 0 & 0 & 0 & 0 & 0 & 0 & 0 & 0 & 0 & 0 & 0 & & 0 & 0 & 0 \\
1 & 0 &. &. &. &. &. &. &. &. &. &. &. & - &. &. &. \\
3 &. & 1 & 1 & 1 & 1 & 1 & 1 & 1 & 1 & 1 & 2 & 1 & - & 1 & 1 & 1 \\
& 2 & 7 & 3 & 8 & 9 & 6 & 7 & 8 & 8 & 9 & 3 & 8 & & 6 & 7 & 8 \\
\hline $\mathrm{C}$ & 0 & 0 & 0 & 0 & 0 & 0 & 0 & 0 & 0 & 0 & 0 & 0 & 0 & & 0 & 0 \\
1 &. &. &. &. &. &. &. &. &. &. &. &. &. & - &. &. \\
4 & 2 & 1 & 1 & 1 & 1 & 1 & 1 & 1 & 1 & 1 & 2 & 1 & 1 & & 1 & 1 \\
& 1 & 7 & 4 & 7 & 6 & 5 & 6 & 8 & 6 & 7 & 3 & 7 & 6 & & 6 & 8 \\
\hline $\mathrm{C}$ & 0 & 0 & 0 & 0 & 0 & 0 & 0 & 0 & 0 & 0 & 0 & 0 & 0 & 0 & & 0 \\
1 &. &. &. &. &. &. &. &. &. &. &. &. &. &. & - &. \\
5 & 2 & 1 & 1 & 1 & 1 & 1 & 1 & 1 & 2 & 2 & 2 & 1 & 1 & 1 & & 1 \\
& 2 & 8 & 5 & 8 & 7 & 6 & 4 & 7 & 1 & 2 & 2 & 8 & 7 & 6 & & 7 \\
\hline $\mathrm{C}$ & 0 & 0 & 0 & 0 & 0 & 0 & 0 & 0 & 0 & 0 & 0 & 0 & 0 & 0 & 0 & \\
1 &. &. &. &. &. &. &. &. &. &. &. &. &. &. &. & - \\
6 & 2 & 1 & 1 & 1 & 1 & 1 & 1 & 1 & 1 & 1 & 2 & 1 & 1 & 1 & 1 & - \\
& 1 & 8 & 6 & 7 & 8 & 8 & 7 & 7 & 8 & 8 & 4 & 7 & 8 & 8 & 7 & \\
\hline
\end{tabular}

\subsubsection{Experiments on Synthetic web Navigational Dataset for SSM-Denclue}

Consider arbitrarily 200 records of web transactions from MSNBC.COM website. The transactions are converted to vector representation, and a $200 \times 200$ similarity matrix is computed using any of the existing similarity measures mentioned above. In the step two after applying SSMDENCLUE clustering technique the clusters formed are 15 .Table $615 \times 15$ matrix which shows the inter cluster distance using Euclidean distance measure.

Table 6: Inter Cluster Distance Using Euclidean Distance for SSM-Denclue

\begin{tabular}{|c|c|c|c|c|c|c|c|c|c|c|c|c|c|c|c|}
\hline & $\mathrm{C}$ & $\mathrm{C}$ & $\mathrm{C}$ & $\mathrm{C}$ & $\mathrm{C}$ & $\mathrm{C}$ & $\mathrm{C}$ & $\mathrm{C}$ & $\mathrm{C}$ & $\mathrm{C}$ & $\mathrm{C}$ & $\mathrm{C}$ & $\mathrm{C}$ & $\mathrm{C}$ & $\mathrm{C}$ \\
& 1 & 2 & 3 & 4 & 5 & 6 & 7 & 8 & 9 & 1 & 1 & 1 & 1 & 1 & 1 \\
& & & & & & & & & 0 & 1 & 2 & 3 & 4 & 5 \\
\hline $\mathrm{C}$ & & 0 & 0 & 0 & 0 & 0 & 0 & 0 & 0 & 0 & 0 & 0 & 0 & 0 & 0 \\
1 & 0 &. &. &. &. &. &. &. &. &. &. &. &. &. &. \\
& & 1 & 1 & 1 & 1 & 1 & 1 & 2 & 1 & 1 & 1 & 1 & 2 & 2 & 2 \\
& & 6 & 7 & 7 & 7 & 8 & 9 & 1 & 9 & 9 & 9 & 9 & & 1 & 2 \\
\hline $\mathrm{C}$ & 0 & & 0 & 0 & 0 & 0 & 0 & 0 & 0 & 0 & 0 & 0 & 0 & 0 & 0 \\
2 &. & 0 &. &. &. &. &. &. &. &. &. &. &. &. &. \\
& 1 & & 1 & 1 & 1 & 1 & 1 & 1 & 1 & 1 & 1 & 1 & 1 & 1 & 1 \\
& 6 & & 7 & 8 & 7 & 7 & 8 & 8 & 7 & 6 & 6 & 6 & 7 & 7 & 8 \\
\hline $\mathrm{C}$ & 0 & 0 & & 0 & 0 & 0 & 0 & 0 & 0 & 0 & 0 & 0 & 0 & 0 & 0 \\
3 &. &. & 0 &. &. &. &. &. &. &. &. &. &. &. &. \\
& 1 & 1 & & 1 & 1 & 1 & 1 & 1 & 1 & 1 & 1 & 1 & 1 & 1 & 1 \\
& 7 & 7 & & 1 & 2 & 3 & 4 & 4 & 4 & 3 & 4 & 3 & 3 & 4 & 5 \\
\hline $\mathrm{C}$ & 0 & 0 & 0 & & 0 & 0 & 0 & 0 & 0 & 0 & 0 & 0 & 0 & 0 & 0 \\
4 &. &. &. & 0 &. &. &. &. &. &. &. &. &. &. &. \\
& 1 & 1 & 1 & & 1 & 1 & 1 & 1 & 1 & 1 & 1 & 1 & 1 & 1 & 1 \\
\hline
\end{tabular}

\begin{tabular}{|c|c|c|c|c|c|c|c|c|c|c|c|c|c|c|c|}
\hline & 7 & 0 & & & & & 4 & & 0 & 2 & 4 & 5 & 5 & 5 & 6 \\
\hline C & 0 & 0 & 0 & 0 & & & 0 & & S & 0 & ) & 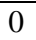 & 0 & 0 & 0 \\
\hline & & & & & 0 & & & & & & & & & & \\
\hline \multirow{2}{*}{5} & 1 & 1 & 1 & 1 & & & 1 & & 1 & 1 & 1 & 1 & 1 & 1 & 1 \\
\hline & 7 & 7 & 2 & 1 & & & 6 & 7 & 7 & 6 & 6 & 6 & 7 & 7 & 8 \\
\hline \multirow[t]{4}{*}{ C } & 0 & 0 & 0 & 0 & 0 & & 0 & 0 & 0 & 0 & 0 & 0 & 0 & 0 & 0 \\
\hline & 1 & $\dot{1}$ & & & & & & & & & & & & & \\
\hline & & 1 & 1 & 1 & 1 & & 1 & 1 & 1 & 1 & 1 & 1 & 1 & 1 & \\
\hline & 8 & 7 & 3 & 2 & 7 & & & 2 & 2 & 3 & 4 & 3 & 3 & 4 & 5 \\
\hline \multirow[t]{4}{*}{$\mathrm{C}$} & 0 & 0 & 0 & 0 & 0 & 0 & & ( & 0 & 0 & 0 & 0 & 0 & 0 & 0 \\
\hline & & 1 & & & & & 0 & & & & & & & & \\
\hline & 1 & 1 & 1 & 1 & 1 & 1 & & 1 & 1 & 1 & 1 & 1 & 2 & 2 & 2 \\
\hline & 9 & & 4 & 4 & 6 & & & & 8 & 9 & 7 & 9 & & 1 & 2 \\
\hline \multirow[t]{3}{*}{ C } & 0 & 0 & 0 & 0 & 0 & 0 & 0 & & 0 & 0 & 0 & 0 & 0 & 0 & 0 \\
\hline & 2 & 1 & 1 & & 1 & & & 0 & & & & & & & 1 \\
\hline & 1 & 8 & 4 & $\epsilon$ & 7 & 2 & 9 & & & 0 & 1 & 1 & 8 & 8 & $\gamma$ \\
\hline \multirow[t]{4}{*}{$\mathrm{C}$} & 0 & 0 & 0 & 0 & 0 & U & 0 & 0 & & 0 & 0 & 0 & 0 & 0 & 0 \\
\hline & & & & & & & & & 0 & & & & & & \\
\hline & 1 & 1 & 1 & 1 & 1 & 1 & & 1 & & & 2 & 1 & 1 & & \\
\hline & 9 & 7 & 4 & 8 & 7 & 2 & 8 & 7 & & & 2 & 7 & 8 & 8 & 2 \\
\hline \multirow{4}{*}{0} & 0 & 0 & 0 & 0 & 0 & 0 & 0 & 0 & 0 & & 0 & 0 & 0 & 0 & 0 \\
\hline & . & & & & & & & & & 0 & & & & & \\
\hline & 1 & 1 & 1 & 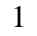 & 1 & 1 & 1 & 1 & 2 & 0 & 1 & 1 & 1 & 1 & 1 \\
\hline & 9 & 6 & 3 & 2 & 6 & 3 & 9 & 6 & & & & 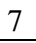 & 8 & 8 & 9 \\
\hline \multirow{4}{*}{$\begin{array}{l}\mathrm{C} \\
1 \\
1\end{array}$} & 0 & 0 & 0 & 0 & 0 & 0 & 0 & 0 & 0 & 0 & & 0 & 0 & 0 & 0 \\
\hline & . & . & & & & & & & & & & & & & \\
\hline & 1 & 1 & 1 & 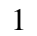 & 1 & 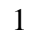 & & 2 & 2 & 1 & & 1 & 1 & 1 & 1 \\
\hline & 9 & 6 & 4 & 4 & 6 & 4 & 1 & 1 & 2 & 7 & & & 4 & 6 & 5 \\
\hline \multirow{4}{*}{$\begin{array}{l}\mathrm{C} \\
1 \\
2\end{array}$} & 0 & 0 & 0 & 0 & 0 & 0 & 0 & 0 & 0 & 0 & 0 & & 0 & 0 & 0 \\
\hline & . & & & & & & & & & & & 0 & & & \\
\hline & 1 & 1 & 1 & 1 & 1 & 1 & 1 & 1 & 1 & 1 & 1 & 0 & 1 & 1 & 1 \\
\hline & 9 & 6 & 3 & 5 & 6 & 3 & & 7 & 7 & 7 & 6 & & 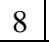 & 7 & 8 \\
\hline \multirow{4}{*}{$\begin{array}{l}\mathrm{C} \\
1 \\
3\end{array}$} & 0 & 0 & 0 & 0 & 0 & 0 & & 0 & 0 & 0 & 0 & 0 & & 0 & 0 \\
\hline & & & & & & & & & & & & & 0 & & \\
\hline & 2 & 1 & 1 & 1 & 1 & 1 & & 1 & 1 & 1 & 1 & 1 & & 1 & 1 \\
\hline & & 7 & 3 & 5 & 1 & $J$ & & 8 & 8 & 8 & 4 & 8 & & 6 & 7 \\
\hline \multirow{4}{*}{$\begin{array}{l}\text { C } \\
1 \\
4\end{array}$} & 0 & 0 & 0 & 0 & 0 & 0 & 0 & 0 & 0 & 0 & 0 & 0 & 0 & & 0 \\
\hline & & & & & & & & & & & & & & & \\
\hline & 2 & 1 & 1 & 1 & 1 & 1 & 2 & 1 & 1 & 1 & 1 & 1 & 1 & 0 & 1 \\
\hline & 1 & 7 & 4 & 5 & 7 & 4 & 1 & 8 & 8 & 8 & 6 & 7 & 6 & & 6 \\
\hline $\mathrm{C}$ & 0 & 0 & 0 & 0 & 0 & 0 & 0 & 0 & 0 & 0 & 0 & 0 & 0 & 0 & \\
\hline & & & & & & & & & & & & & & & 0 \\
\hline & 2 & 1 & 1 & 1 & 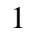 & 1 & 2 & 1 & 2 & 1 & 1 & 1 & 1 & 1 & \\
\hline & 2 & 0 & & 0 & 0 & $J$ & 2 & 0 & 2 & 9 & 5 & & 7 & 6 & \\
\hline
\end{tabular}

\subsubsection{Experiments on Standard web Navigational}

\section{Dataset}

Considered transactions of varying sizes of 5000, $10000,20,000,30000,40000$ from MSNBC dataset. Table 7 shows the number of clusters formed by applying the existing DENCLUE clustering technique and enhanced SSM-DENCLUE. Using the different similarity measures like Euclidean, Jaccard, Cosine, the Inter cluster similarity and Intra cluster similarity are calculated. 
Table 7. Inter and Intra cluster distance for DENCLUE and SSM-DENCLUE

\begin{tabular}{|c|c|c|c|c|c|}
\hline \multicolumn{6}{|c|}{$\begin{array}{c}\text { DENCLUE -CLUSTERING RESULTS USING } \\
\text { EUCLIDEAN }\end{array}$} \\
\hline $\begin{array}{l}\text { No of } \\
\text { Samples }\end{array}$ & 5000 & 10000 & 20000 & 30000 & 40000 \\
\hline $\begin{array}{l}\text { No of } \\
\text { clusters } \\
\text { formed }\end{array}$ & 82 & 124 & 155 & 116 & 189 \\
\hline $\begin{array}{l}\text { Inter } \\
\text { cluster }\end{array}$ & 4.5 & 4.9 & 5.124 & 6.893 & 6.989 \\
\hline $\begin{array}{l}\text { Average } \\
\text { inter } \\
\text { cluster }\end{array}$ & 0.056 & 0.037 & 0.031 & 0.061 & 0.039 \\
\hline $\begin{array}{l}\text { Average } \\
\text { Intra } \\
\text { cluster }\end{array}$ & 4.27 & 4.000 & 4.989 & 6.867 & 5.896 \\
\hline \multicolumn{6}{|c|}{ CLUSTERING RESULTS USING JACCARD } \\
\hline $\begin{array}{l}\text { No of } \\
\text { samples }\end{array}$ & 5000 & 10000 & 20000 & 30000 & 40000 \\
\hline $\begin{array}{l}\text { No of } \\
\text { clusters } \\
\text { formed }\end{array}$ & 99 & 114 & 147 & 135 & 197 \\
\hline $\begin{array}{l}\text { Inter } \\
\text { cluster }\end{array}$ & 4.281 & 4.317 & 5.213 & 8.153 & 7.298 \\
\hline $\begin{array}{l}\text { Average } \\
\text { Inter } \\
\text { cluster }\end{array}$ & 0.043 & 0.037 & 0.035 & 0.060 & 0.037 \\
\hline $\begin{array}{l}\text { Average } \\
\text { Intra } \\
\text { cluster }\end{array}$ & 4.013 & 4.291 & 5.222 & 7.293 & 8.123 \\
\hline \multicolumn{6}{|c|}{ CLUSTERING RESULTS USING COSINE } \\
\hline $\begin{array}{l}\text { No of } \\
\text { samples }\end{array}$ & 5000 & 10000 & 20000 & 30000 & 40000 \\
\hline $\begin{array}{l}\text { No of } \\
\text { clusters } \\
\text { formed }\end{array}$ & 96 & 123 & 156 & 115 & 191 \\
\hline $\begin{array}{l}\text { Inter } \\
\text { cluster }\end{array}$ & 4.6 & 6.367 & 7.214 & 8.135 & 6.721 \\
\hline $\begin{array}{l}\text { Average } \\
\text { Inter } \\
\text { cluster }\end{array}$ & 0.047 & 0.051 & 0.039 & 0.070 & 0.035 \\
\hline $\begin{array}{l}\text { Average } \\
\text { Intra } \\
\text { cluster }\end{array}$ & 4.25 & 4.285 & 6.279 & 7.284 & 6.912 \\
\hline \multicolumn{6}{|c|}{$\begin{array}{c}\text { ( SSM-DENCLUE) CLUSTERING RESULTS } \\
\text { USING SSM }\end{array}$} \\
\hline
\end{tabular}

\begin{tabular}{|l|l|l|l|l|l|}
\hline $\begin{array}{l}\text { Size of } \\
\text { sequences }\end{array}$ & 5000 & 10000 & 20000 & 30000 & 40,000 \\
\hline $\begin{array}{l}\text { No of } \\
\text { clusters }\end{array}$ & 94 & 126 & 149 & 141 & 187 \\
\hline $\begin{array}{l}\text { Inter } \\
\text { cluster }\end{array}$ & 4.681 & 4.317 & 5.213 & 8.153 & 7.298 \\
\hline $\begin{array}{l}\text { Average } \\
\text { Inter } \\
\text { cluster }\end{array}$ & 0.043 & 0.037 & 0.035 & 0.060 & 0.037 \\
\hline $\begin{array}{l}\text { Average } \\
\text { Intra } \\
\text { cluster }\end{array}$ & 4.013 & 4.291 & 5.222 & 7.293 & 8.123 \\
\hline
\end{tabular}

\section{TIME REQUIREMENTS}

Experiments were performed on the above mentioned dataset of varying sizes ,to see the performance of proposed clustering algorithm. The number of clusters formed using DENCLUE for varying sizes of 5000, 10000, 20000, 30000 and 40000 transactions are recorded. The execution time taken for these varying sizes of samples are recorded.

Table 8 Time Requirements of DENCLUE And SSMDENCLUE

\begin{tabular}{|l|l|l|l|l|l|}
\hline \multicolumn{7}{|c|}{ DENCLUE } \\
\hline $\begin{array}{l}\text { Size of } \\
\text { sequences }\end{array}$ & 5000 & 10000 & 20000 & 30000 & 40,000 \\
\hline $\begin{array}{l}\text { No of } \\
\text { clusters }\end{array}$ & 83 & 123 & 156 & 115 & 191 \\
\hline $\begin{array}{l}\text { Time taken } \\
\text { in seconds }\end{array}$ & 1566 & 2665 & 2785 & 3218 & 3196 \\
\hline \multicolumn{5}{|c|}{ SSM-DENCLUE } \\
\hline $\begin{array}{l}\text { Size of } \\
\text { sequences }\end{array}$ & 5000 & 10000 & 20000 & 30000 & 40,000 \\
\hline $\begin{array}{l}\text { No of } \\
\text { clusters }\end{array}$ & 94 & 126 & 149 & 141 & 187 \\
\hline $\begin{array}{l}\text { Time taken } \\
\text { in seconds }\end{array}$ & 1085 & 1879 & 3643 & 1956 & 2498 \\
\hline
\end{tabular}

\section{CONCLUSIONS}

Considered arbitrarily web transactions of equal length from the MSNBC dataset and performed the experiments DENCLUE and SSM-DENCLUE clustering techniques. We used previously existing four different distance/similarity measures namely Euclidean , Jaccard, Cosine, and the newly developed measure called SSM. In DENCLUE the number of clusters are 21,18,18 using Euclidean, Jaccard and Cosine respectively. For good clustering algorithm, the intra cluster distance should be minimum. SSM measure which is a combination of sequence as well as set measure, confirms that the web clustering should consider the sequence as well as set 
value. For example in SSM-DENCLUE for 5000 samples ,the time taken for execution are $1085,1879,3643,1956,2498$ respectively. The time taken to execute the algorithm SSM-DBSCAN is less when compare to other clustering techniques.

A variety of experiments are performed in the context of Density based clustering. A new similarity measure for sequential data $(S S M)$ is devised and incorporated SSM with DENCLUE for Web Usage sequential data. Our results by the way of explanations and list of conclusions, finally showed behavior of clusters that made by enhanced SSMDENCLUE clustering technique on a sequential data in a web usage domain. This new SSM-DENCLUE required less time complexity then the existing.. This experiment shows that, in addition to the content if Sequential Information is also added it improves the quality /accuracy of the clustering. So Sequential information is important as well as Content information is also important

\subsection{Future Work}

we extend our work in future to other clustering techniques and to other domains as well.

- Developing new similarity measures for continuous and discrete sequential data.

- Applying these new clustering techniques to the domains like medical, defense, bioinformatics etc.

- This work can be extended to sequences of unequal length.
- The time complexities of the proposed algorithms can be improved further.

\section{REFERENCES}

[1] Aggarwal.C, Han.J, Wang.J, Yu.P.S, “A Framework for Projected Clustering of High Dimensional Data Streams", Proc. 2004 Int. Conf. on Very Large Data Bases, Toronto, Canada, pp.(852-863), 2004.

[2] Aoying.Z, Shuigeng.Z, "Approaches for scaling DBSCAN algorithm to large spatial database", Journal of Computer Science and Technology, Vol 15(6), pp.(509526), 2000.

[3] Chen Song-Yu, O'Grady2,O'Hare, Wei Wang, "A Clustering Algorithm Incorporating Density and Direction”, IAWTAC ,IEEE 2008.Deepak P, Shourya Roy IBM India Research Lab, OPTICS on Text Data: Experiments and Test Results.

[4] Cooley.R,Mobasher. B,Srivastava.J, "Web mining: Information and pattern discovery on the world wide web", 9th IEEE Int. Conf. Tools AI .

[5] Guha.s, Mishra.n, Motwani.r, Callaghan.1, “ Clustering data streams". In Proceedings of Computer Science. IEEE,November vol.16(10),pp(1391-1399), 2000.

[6] Santhisree, Dr A.Damodaram, 'SSM-DBSCAN and SSM-OPTICS : Incorporating a new similarity measure for Density based Clustering of Web usage data". International Journal on Computer Science and Engineering (IJCSE),Vol.3(9),PP.(3170-3184)September 2011,India. 